\title{
Raman spectroscopy
}

\section{Peter Vandenabeele}

Published online: 12 June 2010

(C) Springer-Verlag 2010

When we look at recent analytical chemistry research papers, it is apparent that Raman spectroscopy is becoming increasingly popular. Several advantageous properties make this technique applicable to a wide range of research projects in fields such as geology, biology, forensics, chemistry, archaeometry, and pharmaceutics. Raman spectroscopy is able to reveal the molecular composition of a sample at the micrometer scale in a nondestructive way.

Although the Raman effect was experimentally demonstrated for the first time in 1928 by the Indian scientist C.V. Raman after it had already been predicted by Smekal, the technique is now coming into vogue. At the time, Sir Raman used filtered sunlight, and he needed several hours of irradiation to be able to record the spectrum of a large volume of a pure liquid. Spectra were recorded on photographic plates. Today, due to instrumental improvements, it is possible to record the spectrum of a micrometersized sample in a couple of seconds.

If we consider the development of Raman spectroscopy, it is clear that it has been instrument-driven. Advances in this field include the introduction of new monochromatic sources of radiation (first mercury lamps and later lasers), new detectors [e.g., charge-coupled device (CCD) detectors], coupling with microscope optics, fiber optics for remote measurements, and FT-Raman spectroscopy. Moreover, the miniaturization of instrumentation has made handheld, battery-powered spectrometers available for infield use, which is very useful for certain applications.

P. Vandenabeele $(\square)$

Department of Archaeology, Ghent University,

Sint-Pietersnieuwstraat 35,

9000 Ghent, Belgium

e-mail: Peter.Vandenabeele@UGent.be
Raman spectroscopy has moved out of specialized laboratories and is increasingly becoming an off-the-shelf technique for the fast and easy characterization of solids or liquids and for quality control. However, to avoid misinterpretations, people still need to be trained to operate the instruments and interpret the spectra obtained by them.

Due to instrumental improvements the technique has become available (and affordable) for use in different research fields, allowing scientists to extract new information from their valued samples. Increased speed of analysis and data processing has also allowed sample mapping, which can provide knowledge on the spatial distribution of the molecules in the sample - a result that is difficult to obtain with other analytical techniques.

In recent years Raman spectroscopy has often been used for analysis in a very wide range of research fields. In microbiology, for instance, the technique can be utilized to record molecular spectra of micrometer-sized samples (e.g., microcolonies of bacteria, fungal spores, etc.). This research can help, for instance, to identify specific species much more quickly than is currently possible, since microcolonies can be grown at a much faster rate than the culture times needed for conventional approaches.

The Raman spectrum of a compound reflects its molecular structure. Though chemically identical, polymorphs with different crystalline structures yield different Raman spectra due to the different local environments of the molecules. The technique can thus be applied to identify mineral phases. In geology, the microanalytical properties of Raman spectroscopy can be used to obtain information on microinclusions, which provide information on the genesis of the rocks containing them. Moreover, since Raman band positions and bandwidths are affected by stresses, the pressure distributions in thin sections of rocks can be discerned. 
In the pharmaceutical industry, Raman spectroscopy is used in quality control: the relatively fast response and the nondestructive nature of the technique allow the products to be analyzed quite easily. Moreover, laser light can be focused through blister packages and inside glass vessels. Handheld instruments that make use of spectral identification algorithms will probably soon be developed to allow customs officers to easily detect counterfeit drugs.

In forensic science, the ability of the technique to penetrate through packages as well as its ability to record spectra from micrometer-sized samples are big advantages. Moreover, as molecular spectra are obtained, the identification of different drugs of abuse is a straightforward task.

Astrobiology is a relatively new research area that attempts to investigate extraterrestrial lifeforms. Among various activities, this involves the development of novel strategies to detect lifeforms if they are present-on Mars for instance. Attempts are being made to identify the "spectroscopic signature of life." Terrestrial lifeforms are being studied in order to elucidate how life can survive under the extreme conditions that are found in space (e.g., how lifeforms can protect themselves against extreme heat or cold, and against extreme UV radiation). Raman spectroscopy is a good candidate technique to use during future missions to Mars in order to detect life. Obviously, before sending a Raman spectrometer to Mars, this approach has to be tested thoroughly.

Raman spectroscopy is also becoming increasingly popular in the field of archaeometry: a research area where techniques from the natural sciences are developed, used and optimized in order to help solve research questions in archaeology, art history and conservation science, among other fields. In this context, the nondestructive character of the technique and the ability to obtain Raman spectra of inorganic as well as organic materials are well appreciated. Moreover, mobile Raman instrumentation allows us to bring the laboratory to the artwork, so that we can investigate precious artworks without the need for sampling.

Technical developments in Raman spectroscopy have always triggered its application in new research fields. We would expect this process to continue: better detectors that can record spectra across a broader spectral range, miniaturization, faster spectral recording, imaging techniques, etc. will probably open up new horizons in the near future. Moreover, the combination of Raman spectroscopy with complementary techniques - such as elemental analysis - will also widen the applicability of the approach. Finally, there is also a trend towards the development of automated spectral identification algorithms, which will make the technique even more accessible to nonspecialist users, although good training in the fundamentals of the technique will always be necessary to avoid misinterpretations.

In this special issue of Analytical and Bioanalytical Chemistry, a series of new and challenging applications of Raman spectroscopy, in various research fields, are presented. This clearly illustrates that - although it is already more than 80 years old-Raman spectroscopy has a sparkling future.

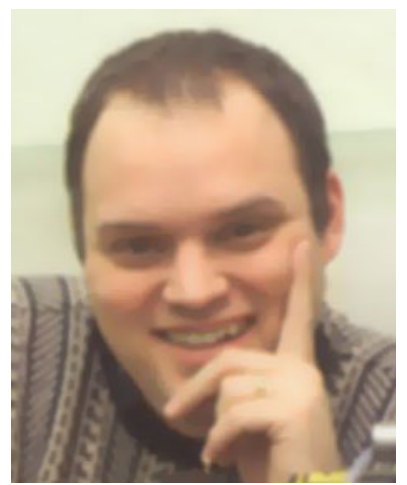

Peter Vandenabeele is research professor in archaeometry at Ghent University. He studied chemistry, focusing in particular on analytical chemistry and Raman spectroscopy. He has authored and co-authored ca. 70 research papers about different applications of Raman spectroscopy, and has given several oral and poster presentations at international conferences. 